\title{
Pb İzotoplarının Alfa Bozunum Yarı-ömürlerinin Farklı Modeller ile İncelenmesi
}

\author{
Fahrettin Koyuncu $^{1 *}(\mathbb{D}$, Asım Soylu² \\ ${ }^{\text {I} B u r d u r ~ M e h m e t ~ A k i f ~ E r s o y ~ U ̈ n i v e r s i t e s i, ~ G o ̈ l h i s a r ~ S a g ̆ l ı k ~ H i z m e t l e r i ~ M Y O, ~ O p t i s y e n l i k ~ P r o g r a m ı, ~ G o ̈ l h i s a r, ~}$ \\ Burdur \\ ${ }^{2}$ Niğde Ömer Halisdemir Üniversitesi, Fen Edebiyat Fakültesi, Fizik Bölümü, Niğde
}

Geliş / Received: 28/05/2019, Kabul / Accepted: 17/08/2020

\begin{abstract}
$\ddot{\mathbf{O} z}$
$\mathrm{Bu}$ çalışmada $\mathrm{Pb}$ izotoplarının $(178 \leq \mathrm{A} \leq 220)$ alfa bozunum yarı-ömürleri, Wentzel-Kramers-Brillion (WKB) metodu, Sahu vd. (2013) formülü ve yakın zamanda önerilen New RenB formülü çerçevesinde incelenmiştir. $\mathrm{Bu}$ üç model alfa bozunumları üzerindeki açısal momentum (L) etkisini içermektedir. Böylelikle farklı mekanizmalar arasında, her bir metot için hesaplanmış rms değerleri kullanılarak karşılaştırmalar da yapılmıştır. Elde edilen sonuçlara göre, değiştirilmiş nükleer yarıçap ifadesine sahip Sahu vd. (2013) formülü, alfa bozunum yarı-ömürlerini açıklamakta oldukça başarılı olmuştur. $\mathrm{Bu}$ çalışmada $\mathrm{Pb}$ izotoplarının alfa bozunum yarı ömürleri hakkında elde edilen tahminler muhtemel deneysel araştırmalara rehberlik edebilir.
\end{abstract}

Anahtar Kelimeler: Alfa bozunumu, Açısal momentum, Pb izotopları, rms.

\section{Investigation of Alpha Decay Half-lives of Pb Isotopes with Different Models}

\begin{abstract}
In this study alpha decay half-lives of $\mathrm{Pb}$ isotopes $(178 \leq \mathrm{A} \leq 220)$ have been investigated in the framework of Wentzel-Kramers-Brillion (WKB) method, Sahu et al. (2013) formula and very recently proposed New RenB formula. These three methods include the angular momentum (L) effects on alpha decay half-lives. In this way comparison between different mechanisms have also been done by using calculated rms values for each method. According to obtained results, Sahu et al. (2013) formula with modified nuclear radius expression has been quite successful to describe the alpha decay half-lives. Obtained predictions on alpha decay half-lives of $\mathrm{Pb}$ isotopes in this study may guide the possible experimental researches.
\end{abstract}

Keywords: Alpha decay, Angular momentum, $\mathrm{Pb}$ isotopes, rms.

\section{Giriş}

Ağır çekirdeklerin bozunumları üzerine yapılan çalışmalar bir süredir nükleer fiziğin ilgi çekici konularındandır. Yüklü parçacık bozunumları; proton, alfa, küme, ağır küme ve kendiliğinden fisyon olmak üzere farklı şekillerde olabilmektedir (Gamow, 1928; Gurney ve Condon, 1928; Bohr ve Wheeler, 1939; Flerov ve Petrzak, 1940; Rose ve Jones,
1984; Price vd., 1985; Barwick vd., 1985). Literatürde yüklü parçacıkların bozunum sürelerini hesaplamakta kullanılan farklı metotlar mevcuttur. Küme ve alfa bozunumlarının yarılanma sürelerinin tahmininde kullanılan; Universal Decay Law (UDL) formülü, Viola-Seaborg-Sobicewski (VSS) yarı ampirik formülü ve Universal Curve (UNIV) bunlardan bazılarıdır (Qi vd., 2009; Viola ve Seaborg, 1966; Poenaru vd., 
2011). Yarı ampirik formüllerin yanı sıra, küme ve alfa bozunumlarının yarılanma sürelerini hesaplamakta kullanılan Coulomb Proximity Potential Model (CPPM) ve WKB de yaygin olarak kullanılan usullerdendir (Blocki vd., 1977; Santosh vd., 2010; Miller ve Good, 1953; Soylu vd., 2018). Zhang ve Wang (2018), Horoi formülü, UDL, UD formülü ve UNIV gibi yaklaşımları kullanarak $Z=118,120$ ve 122 çekirdeklerinin izotopları için küme bozunumlarını araştırmışlardır (Zhang ve Wang, 2018). Santosh vd. (2015), CPPM, UNIV, UDL eşitliklerini ve Genelleştirilmiş Sıvı Damla Modelini (GLDM) kullanarak $\mathrm{Pb}$ izotoplarının $(178 \leq \mathrm{A} \leq 220)$ alfa bozunum süreleri üzerinde tahminlerde bulunmuşlardır. Santosh ve Nithya (2018), Coulomb Proximity Potential Modelin Deforme Çekirdekler (CPPMDN) için uyarlanmış halini, VSS, UDL, Royer ve UNIV eşitliklerini $Z=125$ çekirdeğinin farklı izotoplarının alfa bozunum yarı-ömür sürelerini tahmin etmekte kullanmışlardır. Soylu (2017) Bohr Sommerfeld kuantizasyon şartını WKB modeli ile kullanarak deneysel değerleri var olan bazı küme bozunumları için hesaplamalarda bulunmuştur. Soylu (2017) bu çalışmada fenomonolojik Woods-Saxon (WS), Woods-Saxon kare (WS2) ve mixed Woods-Saxon (mWS) potansiyellerini kullanmıştır. Koyuncu vd. (2017) ${ }^{20} \mathrm{Ne}$ ve ${ }^{44} \mathrm{Ti}$ çekirdeklerinin yapı özelliklerini tespit etmek maksadı ile bu çekirdeklerin alfa bozunum genişliklerinden yararlanmış hesaplamalar için WKB yaklaşımını temel almışlardır.

19. yüzyılın sonlarından itibaren ilgi çekici bir konu olan alfa bozunumu, 1928 yılinda Gamow'un çalışması ve ondan bağımsız olarak Gurney and Condon'un çalışması tarafindan kuantum tünelleme olayı ile açıklanmıştır (Gamow, 1928; Gurney ve Condon, 1928). Yukarıda açıklanmış olan hesaplama yöntemleri gibi, son yillarda ortaya atılan ve Schrödinger denkleminin analitik çözümü ile elde edilen Sahu vd. (2013) denklemi de bu temel vak'a üzerinden hareket etmektedir. Sahu vd. (2013) tarafindan elde edilen bu ilişki Geiger-Nuttall yasasının genelleştirilmiş bir halidir ve açısal momentum taşıyan yüklü parçacıkların bozunum gözlenirlerini açıklamakta kullanılmıştır. Sahu vd. (2013)'nin özgün çalışmasında tüm çekirdekler için nükleer yarıçap (R) $9.5 \mathrm{fm}$ ve preformasyon faktörü $10^{-3}$ seçilmiştir, Zhang vd. (2017) yarıçap ve preformasyon faktörü üzerinde bazı özelleştirmelere giderek Imsahu modelini ortaya koymuştur. VSS yarı ampirik formülünün genelleştirilmesi ile Ren vd. (2004) tarafindan yeni bir ampirik formül önerilmiştir. Ren vd. (2008) bir önceki çalışmaları olan Ren vd. (2004)'de ortaya attıkları formülü WKB bariyer penetrasyon olasılığından yararlanarak ve formüle indirgenmiş kütleyi de ekleyerek yeniden düzenlemişlerdir. $\mathrm{Bu}$ iki çalışmadan yararlanan Akrawy vd. (2019) kisa bir zaman önce izospin ve açısal momentum etkilerini de içerisinde barından iki yeni formül (New RenA ve New RenB) önermişler ve 356 çekirdek için alfa bozunum yarı ömür hesabı yapmışlardır. Bizde bu çalışmalardan yola çıkarak açısal momentumun etkilerini içerisinde barındıran üç farklı yöntemle $\mathrm{Pb}$ izotopları $(178 \leq \mathrm{A} \leq 220)$ için alfa bozunum yarı ömürleri üzerine tahminlerde bulunduk. Hesaplamalarda Sahu, New RenB eşitlikleri ve WKB metodu kullanılmıştır. Sahu vd. (2013) orjinal eşitliğinin kullanılmasının yanı sıra, Zhang vd. (2017) çalışmasına benzer olacak şekilde bir değişikliğe de gidilmiştir. Elde edilen sonuçlar hem deneysel verilerle hem de kendi aralarında kıyaslanmıştır. Hesaplamalar için gerekli olan kodların yazılmasında programlama dili olarak Python 2.7, Spyder 2.0 ara yüzüyle beraber 
kullanılmıştır. $\mathrm{Bu}$ çalışmada kullanılan kodların geliştirilmiş hali olan MES kodu üzerine yapılan çalışmalar halen A. Soylu tarafindan devam ettirilmektedir.

\section{Materyal ve Metot}

$\mathrm{Bu}$ bölümde $\mathrm{Pb}$ izotoplarının alfa bozunum yarı ömürlerini hesaplamakta kullanılan gerekli usullerden bahsedilmiştir. $\mathrm{Pb}$ izotoplarının alfa bozunum yarı ömürlerinin hesaplanmasinda WKB metodu, Sahu vd. (2013) ve New RenB eşitlikleri kullanılmıştır.

\subsection{Sahu eşitliği}

Alfa bozunum yarı ömürlerini tahmin etmede kullandığımız ilk eşitlik Sahu vd. (2013) tarafindan önerilmiştir ve

$$
\log _{10}\left[T_{1 / 2}(s)\right]=a Z_{\alpha} Z_{d} \sqrt{\frac{\mu}{Q_{\alpha}}}+b \sqrt{\mu Z_{\alpha} Z_{d}}+c+d
$$

ile verilir. Burada $T_{1 / 2}(s)$ yarı ömür, $Q_{\alpha}$ bozunum enerjisi, $Z_{\alpha}$ ve $Z_{d}$ sirasiyla alfa ve k1z çekirdeğin proton sayıları ve $\mu=A_{\alpha} A_{d} /\left(A_{a}+A_{d}\right)$ ise indirgenmiş kütledir. $\mathrm{a}, \mathrm{b}, \mathrm{c}, \mathrm{d}$ katsayıları ise şu şekilde verilir

$$
\begin{aligned}
& a=2 a_{0} e^{2} \sqrt{2 m} /(\mathrm{h} \ln 10) \\
& b=-b_{f} \sqrt{2 m e^{2} R} /(\mathrm{h} \ln 10) \\
& c=\ln \left[\left(0.231 x 10^{-23}\right) \sqrt{m R \mu /\left(2 e^{2} Z_{\alpha} Z_{d}\right)} / P\right] / \ln 10 \\
& d=\left[\ln M_{L}-\sum_{l=0}^{L} \ln \left(\eta_{l}\right)\right] / \ln 10 \\
& \text { burada } b_{f}, M_{L} \text { ve } \eta_{l} \\
& b_{f}=2+a_{0}-2 a_{1}+\left(\frac{a_{0}}{4}+a_{1}-2 a_{2}\right) t^{1 / 2} \\
& +\left(\frac{a_{0}}{8}+\frac{a_{1}}{4}+a_{2}-2 a_{3}-1\right) t \\
& +\left(\frac{5 a_{0}}{64}+\frac{a_{1}}{8}+\frac{a_{2}}{4}+a 3\right) t^{3 / 2} \\
& +\left(\frac{5 a_{1}}{64}+\frac{a_{2}}{8}+\frac{a_{3}}{4}-\frac{1}{4}\right) t^{2} \\
& +\left(\frac{5 a_{2}}{64}+\frac{a_{3}}{8}\right) t^{5 / 2}+\left(\frac{5 a_{3}}{64}-\frac{1}{8}\right) t^{3}
\end{aligned}
$$

şeklindedir ve $t=Q_{\alpha} R /\left(e^{2} Z_{\alpha} Z_{d}\right)$ ile verilir. Sahu vd. (2013)'te tüm alfa bozunumları için $R=9.5 \mathrm{fm}$ ve $P=10^{-3}$ olarak seçilmiştir. Sabitler $\quad a_{0}=1.5707288, a_{1}=-0.2121144$, $a_{2}=0.074240 \quad$ son $\quad$ olarak $a_{3}=-0.018729$ şeklinde verilmiştir. Tüm denklemlerde geçerli olmak üzere L açısal momentumu temsil etmektedir (Sahu vd., 2013; Zhang vd., 2017). Bu çalışmada, tüm çekirdekler için kabul edilen $\mathrm{R}=0.95 \mathrm{fm}$ yarıçap değeri, etkileşen çekirdekler cinsinden yazılmış ve $R=1.25\left(A_{d}^{1 / 3}+A_{e}^{1 / 3}\right)$ olarak değiştirilmiştir.

\subsection{New RenB eşitliği}

Hesaplamalarda kullanılan bir diğer yöntem ise Ren vd. (2008) eşitliğini temel alan ve Akrawy vd. (2019) tarafindan önerilen eşitliktir ve

$$
\begin{aligned}
& \log _{10} T_{1 / 2}^{N R B}=a \sqrt{\mu} Z_{1} Z_{2} Q^{-1 / 2}+b \sqrt{\mu Z_{1} Z_{2}} \\
& +c+d I+e I^{2}+f[l(l+1)]
\end{aligned}
$$

ile verilir. Burada $\mu=A_{\alpha} A_{d} /\left(A_{a}+A_{d}\right)$ indirgenmiş kütleyi, $Z_{i}$ alfa ve kız çekirdeğin proton sayılarını, $I=(N-Z) / A$ izospini ve $l$ ise açısal momentumu temsil etmektedir.

Diğer parametreler ise Tablo 1'de verilmiştir.

\subsection{Wentzel-Kramers-Brillouin metodu}

WKB metodu ile Schrödinger dalga denklemi seriye açılarak yaklaşık çözümler elde edilir (Miller ve Good, 1953). WKB nükleer 
reaksiyonların tesir kesitleri, bozunum genişlikleri, yarı ömür hesaplamaları gibi gözlenirleri elde etmede başvurulan yöntemlerden bir tanesidir (Soylu vd., 2018; Soylu, 2017; Koyuncu vd., 2017; Denisov ve Pilipenko, 2012). İkili kümelenme modeline göre etkileşime giren iki çekirdek arasındaki etkin potansiyel

$$
V_{e t}(r)=V_{N}(r)+V_{C}(r)+V_{L}(r)
$$

ile verilir. Burada $V_{N}(r), V_{C}(r)$ ve $V_{L}(r)$ sırasıyla nükleer, Coulomb ve merkezcil potansiyellerdir. Hesaplamalarda Coulomb potansiyeli

$$
\begin{aligned}
& V_{C}(r)=\frac{Z_{d} Z_{\alpha} e^{2}}{r}\left(1-e^{-\varphi r-\frac{1}{2}(\varphi r)^{2}-0.35(\varphi r)^{3}}\right) \\
& \varphi R=\frac{3}{2}
\end{aligned}
$$

şeklinde seçilmiştir. Coulomb potansiyelinin değiştirilmiş bu hali $r=R_{c}$ deki süreksizliği saf dışı bırakmak içindir. Şekli bilinen bir diğer potansiyel ise merkezcil potansiyeldir ve Langer modifiye merkezcil potansiyel

$$
V_{L}(r)=\frac{\mathrm{h}^{2}(L+1 / 2)^{2}}{2 \mu r}
$$

ile verilir. Burada $L$ ve $\mu$ sırasıyla açısal momentum ve indirgenmiş kütledir (Langer, 1937). Etkin potansiyelin geriye kalan son terimi ise $V_{N}(r)$ nükleer potansiyeldir. Nükleer potansiyelin belli bir şekli yoktur ve literatürdeki çalışmalarda kullanılan çeşitli mikroskobik ve fenomonolojik potansiyeller mevcuttur (Buck vd., 1992; Buck vd., 1995; Cook, 1982; Farid vd., 2001; Woods ve Saxon, 1954). Bu çalışmada Woods-Saxon potansiyelinden faydalanılmıştır (Woods ve Saxon, 1954). Bu potansiyelin formu

$$
V_{N}(r)=-\lambda \frac{V_{0}}{\left(1+\exp \left(\frac{r-R}{a}\right)\right)}
$$

şeklindedir ve $\lambda$ normalizasyon parametresi, $V_{0}$ nükleer potansiyelin derinliği, $R$ nükleer yarıçap, $a$ ise difüzyon parametresidir. Hesaplamalarda potansiyelin derinliği $V_{0}=155.3 \mathrm{MeV}$, difüzyon parametresi $a=0.45 \mathrm{fm}$ ve nükleer yarıçap $\mathrm{R}=1.28\left(\left(\mathrm{~A}_{\mathrm{p}}\right)^{1 / 3}+0.8\left(\mathrm{~A}_{p}\right)^{-1 / 3}\right)-0.76 \mathrm{fm} \quad$ olarak seçilmiştir. $A_{p}$ ana çekirdeğinin kütle numarasıdır. Etkin potansiyelin tüm bileşenlerinin tamamlanmasıyla alfa bozunum yarı ömür sürelerinin hesaplanmasına geçilmiştir. Yarılanma süresi

$T_{1 / 2}=\hbar \ln (2) / \Gamma$

ile verilir burada $\Gamma$ bozunum genişliğidir ve

$\Gamma=P_{\alpha} F \frac{\hbar^{2}}{4 \mu} \exp \left[-2 \int_{r_{2}}^{r_{3}} k(r) d r\right]$

ile verilir $\cdot k(r)=\sqrt{\frac{2 \mu}{\hbar^{2}}\left|Q_{\alpha}-V_{e t}(r)\right|}$ dalga sayısını ifade eder, $F$ normalizasyon faktörü iken $P_{\alpha}$ ise preformasyon faktörüdür (Soylu vd., 2018; Soylu, 2017; Koyuncu vd., 2017). Denklem (10)'da verilen $\lambda$ normalizasyon parametresi

$\int_{r_{1}}^{r_{2}} \sqrt{\frac{2 \mu}{\hbar^{2}}\left(Q_{\alpha}-V_{e t}(r)\right)} d r=(G-L+1) \frac{\pi}{2}$

ile verilen Bohr-Sommerfeld Kuantumlanma Şartı ile hesaplanır. Burada $G$ global kuantum numaras1, $Q_{\alpha}$ alfa bozunum enerjisi $\mu$ indirgenmiş kütlesidir. Alfa bozunum enerjisi için üç adet dönüm noktası $\left(r_{1}, r_{2}\right.$ ve $\left.r_{3}\right)$ vardır ve $V_{e t}=Q_{\alpha}$ olduğu noktalarda hesaplanırlar (Soylu vd., 2018; Soylu, 2017; Koyuncu vd., 2017). $G$ global kuantum numaras1; $N<82$ için $G=18, \quad 82 \leq N \leq 126$ için $G=20$ ve $N>126$ için $G=22$ seçilmiştir (Buck vd., 1996). 
Tablo 1. New RenB eşitliğinde kullanılan parametrelerin çekirdek türelerine göre değişimi (Akrawy vd. 2019).

\begin{tabular}{c|cccrrr} 
Çekirdek & $a$ & $b$ & $c$ & $d$ & \multicolumn{1}{c}{$c$} & $f$ \\
Çift-Çift & 0.41107 & -1.44914 & -14.87085 & 13.38618 & -61.47107 & 0 \\
Çift-Tek & 0.44145 & -1.42068 & -16.59713 & -27.68464 & 91.70405 & 0.07947 \\
Tek-Çift & 0.44660 & -1.32208 & -21.09761 & -1.64226 & -17.02692 & 0.07767 \\
Tek-Tek & 0.43323 & -1.40527 & -17.13866 & -7.66291 & 22.26925 & 0.06902 \\
Tüm Ç. & 0.41740 & -1.35824 & -16.86728 & 0.07916 & -16.24873 & 0.09441
\end{tabular}

\section{Bulgular ve Tartışma}

$\mathrm{Bu}$ çalışmada açısal momentum bağımlı üç farklı yöntem ile kurşun izotoplarının alfa bozunum yarı ömürleri hesaplanmıştır. Elde edilen sonuçlar her bir usul için Tablo 2'de verilmiştir. Yarıçap ifadesinde değişiklik yapılmış Sahu vd. (2013) eşitliği Sahu* olarak isimlendirilmiştir. Bunların yanında deneysel verilere en yakın sonuçların hangi model ile elde edildiğini anlamak maksadıyla rms sonuçları

$$
\sigma=\left(\frac{1}{n-1} \sum_{k=1}^{n}\left(\log _{10}\left(T_{\text {hes }}\right)-\log _{10}\left(T_{\text {den }}\right)\right)^{2}\right)^{1 / 2}
$$

denklemi gereğince hesaplanmıştır. Burada $n$ etkileşime giren çekirdek sayısı, $T_{\text {hes }}$ hesaplanan yarılanma ömrü ve $T_{d e n}$ deneysel yarılanma ömrüdür (Soylu ve Koyuncu, 2019). Denklem 14'ten anlaşılacağı üzere bu sonuçlar sadece deneysel verileri var olan alfa bozunumları için gerçekleştirilmiştir. Hesaplamalar sonucu elde edilen rms değerleri Sahu*, Sahu, New RENB ve WKB için sirasiyla $0.876,1.068,1.317$ ve 0.959 olarak elde edilmiştir. $\mathrm{Bu}$ çalışmada kullanılan Q değerleri Santosh vd. (2015)'den alınmıştır.

Tablo 2. $178 \leq \mathrm{A} \leq 220$ Kurşun izotopları için elde edilmiş yarı ömür süreleri ve deneysel değerler. Q bozunum enerjisini ve $\ell$ ise açısal momentumu temsil etmektedir.

\begin{tabular}{ccccccccc}
\hline $\begin{array}{c}\text { Ana } \\
\text { Cekirdek }\end{array}$ & $\begin{array}{c}\mathrm{K} \text { Kz } \\
\text { ekirdek }\end{array}$ & $\begin{array}{c}\mathrm{Q} \\
(\mathrm{MeV})\end{array}$ & $\ell_{\text {min }}$ & $T_{1 / 2}^{\text {Den. }}(s)$ & Sahu* & $\begin{array}{c}\text { Sahu vd. } \\
(2013)\end{array}$ & WKB & $\begin{array}{l}\text { New } \\
\text { Ren B }\end{array}$ \\
\hline${ }^{178} \mathrm{~Pb}$ & ${ }^{174} \mathrm{Hg}$ & 7.824 & 0 & $1.20 \mathrm{E}-04$ & $4.797 \mathrm{E}-04$ & $7.513 \mathrm{E}-05$ & $3.504 \mathrm{E}-04$ & $1.895 \mathrm{E}-04$ \\
${ }^{179} \mathrm{~Pb}$ & ${ }^{175} \mathrm{Hg}$ & 7.629 & 2 & $3.50 \mathrm{E}-03$ & $3.018 \mathrm{E}-03$ & $4.811 \mathrm{E}-04$ & $2.392 \mathrm{E}-03$ & $2.700 \mathrm{E}-03$ \\
${ }^{180} \mathrm{~Pb}$ & ${ }^{176} \mathrm{Hg}$ & 7.452 & 0 & $4.20 \mathrm{E}-03$ & $5.474 \mathrm{E}-03$ & $9.194 \mathrm{E}-04$ & $4.397 \mathrm{E}-03$ & $2.609 \mathrm{E}-03$ \\
${ }^{181} \mathrm{~Pb}$ & ${ }^{177} \mathrm{Hg}$ & 7.269 & 2 & $3.60 \mathrm{E}-02$ & $3.519 \mathrm{E}-02$ & $6.030 \mathrm{E}-03$ & $3.066 \mathrm{E}-02$ & $3.755 \mathrm{E}-02$ \\
${ }^{182} \mathrm{~Pb}$ & ${ }^{178} \mathrm{Hg}$ & 7.099 & 0 & $5.61 \mathrm{E}-02$ & $6.705 \mathrm{E}-02$ & $1.211 \mathrm{E}-02$ & $5.888 \mathrm{E}-02$ & $3.776 \mathrm{E}-02$ \\
${ }^{183} \mathrm{~Pb}$ & ${ }^{179} \mathrm{Hg}$ & 6.962 & 2 & $5.94 \mathrm{E}-01$ & $3.340 \mathrm{E}-01$ & $6.184 \mathrm{E}-02$ & $3.153 \mathrm{E}-01$ & $4.112 \mathrm{E}-01$ \\
${ }^{184} \mathrm{~Pb}$ & ${ }^{180} \mathrm{Hg}$ & 6.807 & 0 & $6.13 \mathrm{E}-01$ & $6.186 \mathrm{E}-01$ & $1.208 \mathrm{E}-01$ & $5.843 \mathrm{E}-01$ & $3.972 \mathrm{E}-01$ \\
${ }^{185} \mathrm{~Pb}$ & ${ }^{181} \mathrm{Hg}$ & 6.729 & 2 & $1.85 \mathrm{E}+01$ & $2.024 \mathrm{E}+00$ & $4.070 \mathrm{E}-01$ & $2.029 \mathrm{E}+00$ & $2.767 \mathrm{E}+00$ \\
${ }^{186} \mathrm{~Pb}$ & ${ }^{182} \mathrm{Hg}$ & 6.504 & 0 & $1.21 \mathrm{E}+01$ & $7.431 \mathrm{E}+00$ & $1.570 \mathrm{E}+00$ & $7.566 \mathrm{E}+00$ & $5.402 \mathrm{E}+00$ \\
${ }^{187} \mathrm{~Pb}$ & ${ }^{183} \mathrm{Hg}$ & 6.427 & 7 & $1.53 \mathrm{E}+02$ & $1.296 \mathrm{E}+03$ & $2.626 \mathrm{E}+02$ & $4.683 \mathrm{E}+03$ & $2.051 \mathrm{E}+06$ \\
${ }^{188} \mathrm{~Pb}$ & ${ }^{184} \mathrm{Hg}$ & 6.143 & 0 & $2.70 \mathrm{E}+02$ & $1.905 \mathrm{E}+02$ & $4.347 \mathrm{E}+01$ & $2.118 \mathrm{E}+02$ & $1.580 \mathrm{E}+02$ \\
${ }^{189} \mathrm{~Pb}$ & ${ }^{185} \mathrm{Hg}$ & 5.905 & 2 & $3.90 \mathrm{E}+03$ & $3.156 \mathrm{E}+03$ & $8.219 \mathrm{E}+02$ & $4.345 \mathrm{E}+03$ & $6.470 \mathrm{E}+03$ \\
${ }^{190} \mathrm{~Pb}$ & ${ }^{186} \mathrm{Hg}$ & 5.732 & 0 & $1.78 \mathrm{E}+04$ & $1.161 \mathrm{E}+04$ & $2.860 \mathrm{E}+03$ & $1.426 \mathrm{E}+04$ & $1.098 \mathrm{E}+04$ \\
${ }^{191} \mathrm{~Pb}$ & ${ }^{187} \mathrm{Hg}$ & 5.487 & 0 & $7.98 \mathrm{E}+05$ & $1.719 \mathrm{E}+05$ & $4.395 \mathrm{E}+04$ & $2.237 \mathrm{E}+05$ & $1.740 \mathrm{E}+05$ \\
${ }^{192} \mathrm{~Pb}$ & ${ }^{188} \mathrm{Hg}$ & 5.255 & 0 & $3.56 \mathrm{E}+06$ & $2.648 \mathrm{E}+06$ & $7.035 \mathrm{E}+05$ & $3.638 \mathrm{E}+06$ & $2.841 \mathrm{E}+06$ \\
${ }^{193} \mathrm{~Pb}$ & ${ }^{189} \mathrm{Hg}$ & 5.049 & 0 & & $3.532 \mathrm{E}+07$ & $9.767 \mathrm{E}+06$ & $5.092 \mathrm{E}+07$ & $3.965 \mathrm{E}+07$ \\
\hline
\end{tabular}




\begin{tabular}{|c|c|c|c|c|c|c|c|c|}
\hline${ }^{194} \mathrm{~Pb}$ & ${ }^{190} \mathrm{Hg}$ & 4.772 & 0 & $8.80 \mathrm{E}+09$ & $1.545 \mathrm{E}+09$ & $4.437 \mathrm{E}+08$ & $2.373 \mathrm{E}+09$ & $1.826 \mathrm{E}+09$ \\
\hline${ }^{195} \mathrm{~Pb}$ & ${ }^{191} \mathrm{Hg}$ & 4.489 & 0 & & $1.070 \mathrm{E}+11$ & $3.194 \mathrm{E}+10$ & $1.753 \mathrm{E}+11$ & $1.317 \mathrm{E}+11$ \\
\hline${ }^{196} \mathrm{~Pb}$ & ${ }^{192} \mathrm{Hg}$ & 4.259 & 0 & $7.40 \mathrm{E}+09$ & $4.594 \mathrm{E}+12$ & $1.428 \mathrm{E}+12$ & $7.923 \mathrm{E}+12$ & $5.775 \mathrm{E}+12$ \\
\hline${ }^{197} \mathrm{~Pb}$ & ${ }^{193} \mathrm{Hg}$ & 3.923 & 0 & & $2.101 \mathrm{E}+15$ & $6.785 E+14$ & $3.903 \mathrm{E}+15$ & $2.677 \mathrm{E}+15$ \\
\hline${ }^{198} \mathrm{~Pb}$ & ${ }^{194} \mathrm{Hg}$ & 3.743 & 0 & & $7.764 \mathrm{E}+16$ & $2.618 \mathrm{E}+16$ & $1.500 \mathrm{E}+17$ & $9.835 E+16$ \\
\hline${ }^{199} \mathrm{~Pb}$ & ${ }^{195} \mathrm{Hg}$ & 3.377 & 2 & & $5.579 \mathrm{E}+20$ & $1.939 \mathrm{E}+20$ & $1.254 \mathrm{E}+21$ & $1.400 \mathrm{E}+21$ \\
\hline${ }^{200} \mathrm{~Pb}$ & ${ }^{196} \mathrm{Hg}$ & 3.185 & 0 & & $4.212 \mathrm{E}+22$ & $1.542 \mathrm{E}+22$ & $9.170 \mathrm{E}+22$ & $4.954 \mathrm{E}+22$ \\
\hline${ }^{201} \mathrm{~Pb}$ & ${ }^{197} \mathrm{Hg}$ & 2.891 & 2 & & $3.719 \mathrm{E}+26$ & $1.409 \mathrm{E}+26$ & $9.304 E+26$ & $8.241 E+26$ \\
\hline${ }^{202} \mathrm{~Pb}$ & ${ }^{198} \mathrm{Hg}$ & 2.624 & 0 & & $1.604 \mathrm{E}+30$ & $6.390 \mathrm{E}+29$ & $3.923 \mathrm{E}+30$ & $1.544 \mathrm{E}+30$ \\
\hline${ }^{203} \mathrm{~Pb}$ & ${ }^{199} \mathrm{Hg}$ & 2.370 & 2 & & $5.844 \mathrm{E}+34$ & $2.417 \mathrm{E}+34$ & $1.635 \mathrm{E}+35$ & $9.920 \mathrm{E}+34$ \\
\hline${ }^{204} \mathrm{~Pb}$ & ${ }^{200} \mathrm{Hg}$ & 2.004 & 0 & & $1.383 \mathrm{E}+42$ & $6.004 E+41$ & $3.829 \mathrm{E}+42$ & $8.394 \mathrm{E}+41$ \\
\hline${ }^{205} \mathrm{~Pb}$ & ${ }^{201} \mathrm{Hg}$ & 1.502 & 2 & & $1.483 \mathrm{E}+57$ & $6.666 \mathrm{E}+56$ & $4.948 \mathrm{E}+57$ & $9.069 \mathrm{E}+56$ \\
\hline${ }^{206} \mathrm{~Pb}$ & ${ }^{202} \mathrm{Hg}$ & 1.170 & 0 & & $3.969 \mathrm{E}+71$ & $1.876 \mathrm{E}+71$ & $1.286 \mathrm{E}+72$ & $5.327 \mathrm{E}+70$ \\
\hline${ }^{207} \mathrm{~Pb}$ & ${ }^{203} \mathrm{Hg}$ & 0.427 & 2 & & $1.436 \mathrm{E}+154$ & $7.019 \mathrm{E}+153$ & $5.847 \mathrm{E}+154$ & $2.547 \mathrm{E}+151$ \\
\hline${ }^{208} \mathrm{~Pb}$ & ${ }^{204} \mathrm{Hg}$ & 0.552 & 0 & & $6.446 \mathrm{E}+128$ & $3.338 \mathrm{E}+128$ & $2.329 E+129$ & $2.709 \mathrm{E}+126$ \\
\hline${ }^{209} \mathrm{~Pb}$ & ${ }^{205} \mathrm{Hg}$ & 2.282 & 5 & & $1.868 \mathrm{E}+37$ & $1.021 \mathrm{E}+37$ & $5.763 \mathrm{E}+37$ & $5.844 E+38$ \\
\hline${ }^{210} \mathrm{~Pb}$ & ${ }^{206} \mathrm{Hg}$ & 3.826 & 0 & & $8.275 E+15$ & $4.943 \mathrm{E}+15$ & $1.119 \mathrm{E}+16$ & $9.342 \mathrm{E}+15$ \\
\hline${ }^{211} \mathrm{~Pb}$ & ${ }^{207} \mathrm{Hg}$ & 3.605 & 0 & $3.69 \mathrm{E}+16$ & $8.786 \mathrm{E}+17$ & $5.481 \mathrm{E}+17$ & $1.247 \mathrm{E}+18$ & $9.728 \mathrm{E}+17$ \\
\hline${ }^{212} \mathrm{~Pb}$ & ${ }^{208} \mathrm{Hg}$ & 3.332 & 0 & & $5.432 \mathrm{E}+20$ & $3.539 \mathrm{E}+20$ & $8.172 E+20$ & $5.775 \mathrm{E}+20$ \\
\hline${ }^{213} \mathrm{~Pb}$ & ${ }^{209} \mathrm{Hg}$ & 3.047 & 0 & & $1.134 \mathrm{E}+24$ & $7.714 \mathrm{E}+23$ & $1.810 \mathrm{E}+24$ & $1.124 \mathrm{E}+24$ \\
\hline${ }^{214} \mathrm{~Pb}$ & ${ }^{210} \mathrm{Hg}$ & 2.799 & 0 & & $2.269 \mathrm{E}+27$ & $1.615 E+27$ & $3.815 E+27$ & $2.054 \mathrm{E}+27$ \\
\hline${ }^{215} \mathrm{~Pb}$ & ${ }^{211} \mathrm{Hg}$ & 2.649 & 0 & & $3.739 \mathrm{E}+29$ & $2.784 \mathrm{E}+29$ & $6.484 E+29$ & $3.133 E+29$ \\
\hline${ }^{216} \mathrm{~Pb}$ & ${ }^{212} \mathrm{Hg}$ & 2.329 & 0 & & $1.091 \mathrm{E}+35$ & $8.499 \mathrm{E}+34$ & $2.018 \mathrm{E}+35$ & $7.526 \mathrm{E}+34$ \\
\hline${ }^{217} \mathrm{~Pb}$ & ${ }^{213} \mathrm{Hg}$ & 2.179 & 0 & & $1.008 \mathrm{E}+38$ & $8.219 E+37$ & $1.921 \mathrm{E}+38$ & $6.116 \mathrm{E}+37$ \\
\hline${ }^{218} \mathrm{~Pb}$ & ${ }^{214} \mathrm{Hg}$ & 1.879 & 0 & & $1.011 \mathrm{E}+45$ & $8.632 E+44$ & $2.043 \mathrm{E}+45$ & $4.501 \mathrm{E}+44$ \\
\hline${ }^{219} \mathrm{~Pb}$ & ${ }^{215} \mathrm{Hg}$ & 1.679 & 0 & & $4.898 \mathrm{E}+50$ & $4.383 \mathrm{E}+50$ & $1.029 \mathrm{E}+51$ & $1.648 \mathrm{E}+50$ \\
\hline${ }^{220} \mathrm{~Pb}$ & ${ }^{216} \mathrm{Hg}$ & 1.419 & 0 & & $6.855 E+59$ & $6.427 \mathrm{E}+59$ & $1.513 \mathrm{E}+60$ & $1.432 \mathrm{E}+59$ \\
\hline
\end{tabular}

\section{Sonuç ve Öneriler}

Bozunum esnasında alfa parçacığının taşıdığı açısal momentumun yarı ömür süreleri üzerindeki önemli bir etkisi olduğu bilinmektedir. Açısal momentum bağımlı üç farklı model kullanılarak elde edilen sonuçlara göre Sahu* formülünün diğer model ve formüllere göre deneysel verilere daha yakın olduğu rms değerlerine dayanarak söylenebilir. Açısal momentum bağımlı bu üç mekanizma arasında $\mathrm{L}=0$ değeri için $\mathrm{WKB}$ yönteminde katkı olduğu görülebilir. WKB metodunda kullanılan Langer modifiye merkezcil potansiyel $\mathrm{L}=0$ için bile bariyere neden olur. Açısal momentum etkin potansiyelin bir parçası olduğu için bu terim bir ek katk1 getirir. Bu katk1 efektif potansiyelin şeklinde değişikliğe neden olur ve bunun dönüm noktalarını etkilemesi beklenir. Denklem (12) ve (13) gereğince dönüm noktaları bütün sistem üzerinde önemli bir yere sahiptir ve sonucu doğrudan etkileyerek alfa bozunum sürelerini değiştirir. $\mathrm{O}$ halde herhangi bir bozunum için açısal momentumun yarı ömürlere olan katkısını incelemek elde edilecek sonuçlar için gayet mühimdir.

Diğer yandan Akrawy vd. (2019) tarafından geliştirilen. Genel olarak New RenB formülü ile elde edilen sonuçlar gözden geçirildiğinde açisal momentumu $l_{\min }=0$ ve 2 olan çekirdekler için sonuçların (deneysel verileri hali hazırda bulunan alfa bozunumları göz önüne alınmıştır) deneysel veriler ile gayet uyumlu olduğu söylenebilir. Ancak ${ }^{187} \mathrm{~Pb} \rightarrow$ ${ }^{183} \mathrm{Hg}+\alpha$ bozunumu için $l_{\min }=7$ dir, bu bozunum için deneysel sonuç $1.53 \mathrm{E}+02$ iken New RenB ile elde edilen sonuç $2.051 \mathrm{E}+06$ 'dır. $\mathrm{Bu}$ sonuç New RENB için elde edilen rms değerinin diğer model ve 
eşitliklere göre yüksek çıkmasına neden olmuştur. Bu sebepten dolayı, New RenB eşitliği rms verilerine göre deneysel verilere uygunluk bakımından en uzak sonuçları üretmiştir. Santosh vd. (2015) ise $\mathrm{Pb}$ izotopları üzerine yaptıkları hesaplamalar sonucu rms değerlerini CPPM, UNIV, UDL ve GLDM için sırasıyla 1.061, 1.012, 1.219 ve 0.897 olarak rapor etmişlerdir. Dolayısıyla bu çalışmada elde edilen Sahu* ve WKB rms değerlerinin Santosh vd. (2015)'de elde edilenlere göre düşük olması deneysel değerlere uygunluk açısından önem arz etmektedir.

Sahu vd. (2013) eşitliğinde Zhang vd. (2017)'ye benzer şekilde yapılan değişiklik ile nükleer yarıçapın bozunum sonucu ortaya çıkan parçacıklar cinsinden yazılmasının etkileri de araştırılmıştır. Elde edilen sonuçlar yarıçapta yapılan ufak bir düzeltmenin bile sonuçları olumlu etkilediğini göstermiştir. Zhang vd. (2017)'de hem nükleer yarıçap hem de preformasyon faktörü üzerine yapılan değişikliklerin sonuçları ne denli değiştirdiği tartışılmıştı. Dolayısıyla Sahu vd. (2013) formülü üzerinde yapılacak değişiklikler muhtemel yeni çalışmalara kapı açabilecek niteliktedir. Ayrıca bu çalışma ile elde edilen sonuçların yapılması muhtemel deneysel çalışmalara yol gösterici nitelikte olduğu düşünülmektedir.

\section{Teşekkür}

Bu çalışma 118R028 numaralı TÜBİTAK projesi ile desteklenmiştir.

\section{Kaynaklar}

Gamow, G. 1928. "Zur quantentheorie des atomkernes", Zeitschrift für Physik, 51:(3-4), 204-212.

Gurney, R. W., \& Condon, E. U. 1928. "Wave mechanics and radioactive disintegration", Nature, 122:(3073), 439.

Bohr, N., \& Wheeler, J. A. 1939. "The mechanism of nuclear fission", Physical Review, 56:(5), 426.

Flerov, G. N., \& Petrzhak, K. A. 1940. "Spontaneous fission of uranium", Physical Review, 58:(89), 275-279.

Rose, H. J., \& Jones, G. A. 1984. “A new kind of natural radioactivity”, Nature, 07:(5948), 245.

Price, P. B., Stevenson, J. D., Barwick, S. W., \& Ravn, H. L. 1985. "Discovery of Radioactive Decay of $\mathrm{Ra}^{222}$ and $\mathrm{Ra}^{224}$ by $\mathrm{C}^{14}$ Emission", Physical review letters, 54:(4), 297.
Barwick, S. W., Price, P. B., \& Stevenson, J. D. 1985. "Radioactive decay of $\mathrm{U}^{232}$ by $\mathrm{Ne}^{24}$ emission", Physical Review C, 31:(5), 1984.

Qi, C., Xu, F. R., Liotta, R. J., \& Wyss, R. 2009. "Universal decay law in charged-article emission and exotic cluster radioactivity", Physical review letters, 103:(7), 072501.

Viola Jr, V. E., \& Seaborg, G. T. 1966. "Nuclear systematics of the heavy elements-II Lifetimes for alpha, beta and spontaneous fission decay" Journal of Inorganic and Nuclear Chemistry, 28:(3), 741-761.

Poenaru, D. N., Gherghescu, R. A., \& Greiner, W. 2011. "Single universal curve for cluster radioactivities and $\alpha$ decay", Physical Review C, 83:(1), 014601.

Błocki, J., Randrup, J., Światecki, W. J., \& Tsang, C. F. 1977. "Proximity forces", Annals of Physics, 105:(2), 427-462.

Santhosh, K. P., Biju, R. K., \& Sahadevan, S. 2010. "Semi-empirical formula for 
spontaneous fission half life time", Nuclear Physics A, 832:(3-4), 220-232.

Miller Jr, S. C., \& Good Jr, R. H. 1953. "A WKB-type approximation to the Schrödinger equation", Physical Review, 91:(1), 174.

Soylu, A., Koyuncu, F., Coban, A., Bayrak, O., \& Freer, M. 2018. "Investigation of deformation effects on the decay properties of ${ }^{12} \mathrm{C}+\alpha$ Cluster states in ${ }^{16} \mathrm{O}$ ", Annals of Physics, 391, 263-277.

Zhang, Y. L., \& Wang, Y. Z. 2018. "Systematic study of cluster radioactivity of superheavy nuclei”, Physical Review $C$, 97:(1), 014318.

Santhosh, K. P., Sukumaran, I., \& Priyanka, B. 2015. "Theoretical studies on the alpha decay of $178-220 \mathrm{~Pb}$ isotopes", Nuclear Physics A, 935, 28-42.

Santhosh, K. P., \& Nithya, C. 2018. " $\alpha$-decay chains of superheavy nuclei with $\mathrm{Z}=125$ ", Physical Review C, 97(4), 044615.

Soylu, A. 2017. Phenomenological potential calculations for cluster decays. Turkish Journal of Physics, 41:(3), 217-226.

Koyuncu, F., Soylu, A., \& Bayrak, O. 2017. "Binary cluster model calculations for ${ }^{20} \mathrm{Ne}$ and ${ }^{44}$ Ti nuclei", Modern Physics Letters A, 32:(09), 1750050.

Sahu, B., Paira, R., \& Rath, B. 2013. "General decay law for emission of charged particles and exotic cluster radioactivity", Nuclear Physics A, 908, 40-50.

Zhang, S., Zhang, Y., Cui, J., \& Wang, Y. 2017. "Improved semi-empirical relationship for $\alpha$-decay half-lives", Physical Review C, 95:(1), 014311.

Ren, Z., Xu, C., \& Wang, Z. 2004. "New perspective on complex cluster radioactivity of heavy nuclei”, Physical Review C, 70:(3), 034304.

Ni, D., Ren, Z., Dong, T., \& Xu, C. 2008. "Unified formula of half-lives for $\alpha$ decay and cluster radioactivity", Physical Review $C$, 78:(4), 044310.

Akrawy, D. T., Hassanabadi, H., Hosseini, S. S., \& Santhosh, K. P. 2019. "Influence of nuclear isospin and angular momentum on alpha-decay half-lives", Nuclear Physics A, 983, 310-320.

Denisov, V. Y., \& Pilipenko, N. A. (2010). "Fusion of deformed nuclei: C 12+C12". Physical Review C, 81(2), 025805.

Langer, R. E. 1937. "On the connection formulas and the solutions of the wave equation", Physical Review, 51:(8), 669.

Buck, B., Merchant, A. C., \& Perez, S. M. 1992. " $\alpha$ decay calculations with a realistic potential”, Physical Review C, 45:(5), 2247.

Buck, B., Johnston, J. C., Merchant, A. C., \& Perez, S. M. 1995. "Unified treatment of scattering and cluster structure in $\alpha+$ closed shell nuclei: $\mathrm{Ne}^{20}$ and $\mathrm{Ti}^{44}$ ", Physical Review C, 52:(4), 1840.

Cook, J. 1982. "Double-folding model analysis of deuteron elastic scattering", Nuclear Physics A 382:(1) 61-70.

Farid, M. E. A., Mahmoud, Z. M. M., \& Hassan, G. S. 2001. "Analysis of heavy ions elastic scattering using the double folding cluster model", Nuclear Physics A, 691:(3-4), 671-690.

Woods, R. D., \& Saxon, D. S. 1954. "Diffuse surface optical model for nucleon-nuclei scattering", Physical Review, 95:(2), 577.

Buck, B., Merchant, A. C., \& Perez, S. M. 1996. "Exotic cluster states in actinide nuclei”, Physical review letters, 76:(3), 380.

Soylu, A., \& Koyuncu, F. 2019. "The predictions on the heavier cluster decays of superheavy nuclei", The European Physical Journal A, 55(7), 118. 